\title{
Intracranial Metaplastic Meningioma : Clinical and Radio- logical Characteristics of 11 Cases
}

\author{
Taehoon Kim, ${ }^{1}$ Jin Wook Kim, ${ }^{1}$ So Young Ji, ${ }^{1}$ Ho Kang, ${ }^{1}$ Kyung-Min Kim, ${ }^{1}$ Yong Hwy Kim, Chul-Kee Park, ${ }^{1}$ Seung Hong Choi, ${ }^{2}$ \\ Sung-Hye Park ${ }^{3}$ \\ Department of Neurosurgery, Seoul National University Hospital, Seoul, Korea \\ Department of Radiology, ${ }^{2}$ Seoul National University Hospital, Seoul, Korea \\ Department of Pathology, ${ }^{3}$ Seoul National University Hospital, Seoul, Korea
}

Objective : Metaplastic meningioma is an extremely rare subtype of World Health Organization (WHO) grade I meningioma. It has distinctive histological subtypes according to its own mesenchymal components. Owing to its scarcity, clinical or radiological features of a metaplastic meningioma are poorly described.

Methods : Between 2004 and 2018, we analyzed total 1814 cases surgically proven meningioma for 15 years. Among them, metaplastic meningioma was diagnosed in 11 cases. Magnetic resonance images were taken for all patients, and computed tomography scan was taken for 10 patients.

Results : WHO grade I meningiomas were 1376 cases (75.9\%), 354 cases (19.5\%) in WHO grade II, and 84 cases (4.6\%) in WHO grade III meningiomas. Metaplastic meningioma was 11 cases as $0.8 \%$ of WHO grade I meningioma and $0.6 \%$ of entire meningiomas for 15 years. Among the entire 11 metaplastic meningiomas, five tumors (45\%) were diagnosed as a lipomatous subtype with rich fat components, four (36\%) as an osseous subtype with extensive bone formation and two (18\%) as a xanthomatous subtype. There was no cartilaginous subtype metaplastic meningioma in our study. Lipomatous and osseous metaplastic meningioma have peculiar radiological characteristics according to mesenchymal components.

Conclusion : We investigated a rare metaplastic meningioma subtype based on our 15-year surgical experience with meningiomas. Further investigation will be necessary for the clear clarification of tumor nature of this rare tumor.

Key Words : Meningioma of metaplasia · Magnetic resonance imaging · Lipomatous.

\section{INTRODUCTION}

World Health Organization (WHO) grade I benign meningioma includes nine subtypes : meningothelial, fibrous, transitional, psammomatous, angiomatous, microcystic, secretory, lymphoplasmacyte-rich, and metaplastic meningioma ${ }^{1,5,6,8,9,19,21)}$. Among these, metaplastic meningioma is an extremely rare subtype, defined as containing focal or widespread mesenchymal components including osseous, cartilaginous, lipomatous, and myxoid or xanthomatous tissue, singly or in combina-

- Received : May 27, 2020 •Revised : July 7, 2020 •Accepted : July 8, 2020

- Address for reprints : Jin Wook Kim

Department of Neurosurgery, Seoul National University Hospital, 101 Daehak-ro, Jongno-gu, Seoul 03080, Korea

Tel : +82-2-2072-2862, Fax : +82-2-744-8459, E-mail : wook616@hanmail.net, ORCID : https://orcid.org/0000-0002-3773-3940

This is an Open Access article distributed under the terms of the Creative Commons Attribution Non-Commercial License (http://creativecommons.org/licenses/by-nc/4.0) which permits unrestricted non-commercial use, distribution, and reproduction in any medium, provided the original work is properly cited. 
$\operatorname{tion}^{1,2)}$. In addition, metaplastic meningiomas are often referred to as lipomatous, osseous or xanthomatous, based on their dominant mesenchymal components ${ }^{19)}$.

The clinical and radiological characteristics of meningiomas, the most common extraaxial primary brain tumor, have been widely described. The unique radiological features associated with their histology have also been delineated. Microcystic meningioma, characterized by the formation of intercellular microcysts, show the distinguishing radiological feature of markedly high signal intensity with severe peritumoral edema in T2-weighted images ${ }^{4,13,16)}$. Sclerosing meningioma also show peculiar radiological features, such as very low signal intensity on T2-weighted images due to their abundant calcification ${ }^{10}$. Secretory meningioma are characterized by an intracellular lumen containing an eosinophilic secretion (i.e., a pseudopsammoma body), which shows a characteristic high intensity in up to $96 \%$ of cases. They may also exhibit relatively high apparent diffusion coefficient values compared with typical meningiomas ${ }^{11,13}$.

Due to their rarity, the clinical and radiological features of metaplastic meningiomas have been poorly described. Thus, the study goal was to systematically review all available surgically confirmed metaplastic meningiomas at our center, to determine whether they have unique clinical and/or radiological characteristics.

\section{MATERIALS AND METHODS}

This study was performed in accordance with the ethical standards of the International Research Board of Seoul National University (IRB No. H-2005-163-1125). We retrospectively analyzed patients whose diagnosis with meningioma was surgically confirmed at our institute between 2004 and 2018, during which time 1814 total cases were identified. Eleven consecutive cases were found to have been pathologically confirmed as metaplastic meningioma. These patients' clinical, radiological, histopathological, and surgical records were obtained from electronic databases and reviewed in detail. These patients (four males and seven females) had a mean age of 60 years.

For radiological analyses, preoperative magnetic resonance (MR) and computed tomography (CT) images were collected, including: attenuation (high, iso-dense, low), calcification, and fat component (absent versus present) for CT images; T1and T2-weighted signal intensity (high, iso, or low), enhancement pattern (homogeneous versus heterogeneous), and peritumoral edema (absent versus present) were collected for MR images. Histopathological reviews were repeated by a neuropathologist.

\section{RESULTS}

From 2004 to 2018, a total of 1814 surgically confirmed meningioma cases were seen at our institute. Metaplastic meningioma was diagnosed in 11 cases, representing $0.8 \%$ of WHO grade I meningioma and $0.6 \%$ of all meningiomas during the 15 -year study period. The details of these meningioma grades and subtypes are shown in Fig. 1.

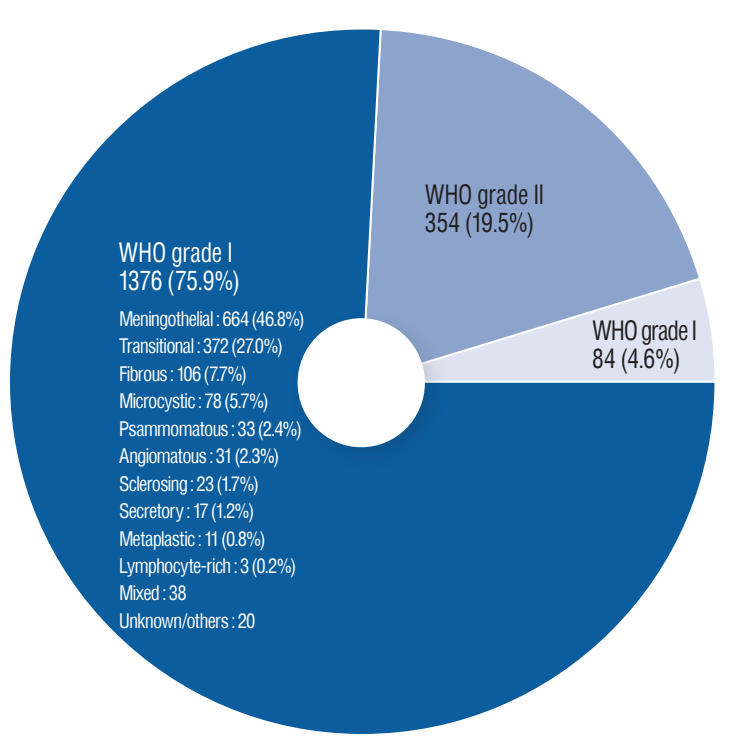

Fig. 1. Distribution of surgically proven meningioma cases from 2004 to 2018 according to WHO grade. There were 1376 (75.9\%) WHO grade I benign meningioma cases; 354 (19.5\%) and 84 cases (4.6\%) were WHO grades II and III, respectively. Among the WHO grade I meningioma, meningothelial subtype was the most common, occurring in 644 of 1376 cases $(46.8 \%)$. The second most common subtype was transitional meningioma (372 cases, $27.0 \%$ ), followed by fibrous, microcystic, and psammomatous subtypes. Relatively rare with less than $2 \%$ incidence were sclerosing, secretory, metaplastic and lymphocyte-rich meningiomas. Metaplastic meningioma was diagnosed in 11 cases, representing $0.8 \%$ of WHO grade I meningioma and $0.6 \%$ of all meningiomas during the 15 -year study period. The rarest was lymphocyte-rich subtype, with only three cases (0.2\%). WHO : World Health Organization. 


\section{Illustrative case 1}

A 54-year-old woman visited the outpatient clinic for evaluation of a brain tumor that had been incidentally detected following a traffic accident. She had no neurological symptoms or signs. Brain CT showed a $4.8 \mathrm{~cm}$ heterogeneously isodense lesion surrounded by a hypodense edge on the left frontal convexity and a $3.4 \mathrm{~cm}$ bony mass with ground-glass opacity on the left frontal bone. MR images revealed a $4.8 \mathrm{~cm}$ mass with heterogeneous isosignal intensity in T2-weighted images suggesting the involvement of fatty components. The mass appeared as a heterogeneously enhancing lesion after contrast administration. Despite the tumor size, definite peritumoral edema was absent. The primary preoperative radiological diagnosis was meningioma with teratoma or fatty components, though teratoma could not be excluded (Fig. 2A-D).

After a typical frontal craniotomy, a grey-whitish hard mass was visualized and internal debulking was accomplished with a Cavitron ultrasonic surgical aspirator. The tumor was completely removed after extracapsular dissection superiorly, anteriorly, and medially. The patient was discharged without neurological deterioration.

Upon microscopic examination, the mass was a poorly cir- cumscribed lesion with multinodular lobular architecture. The tumor cells had abundant eosinophilic cytoplasm and uniform, round-to-oval nuclei with minimal cytology atypia. Whorl formation was frequently observed. Adipocytic prolif-
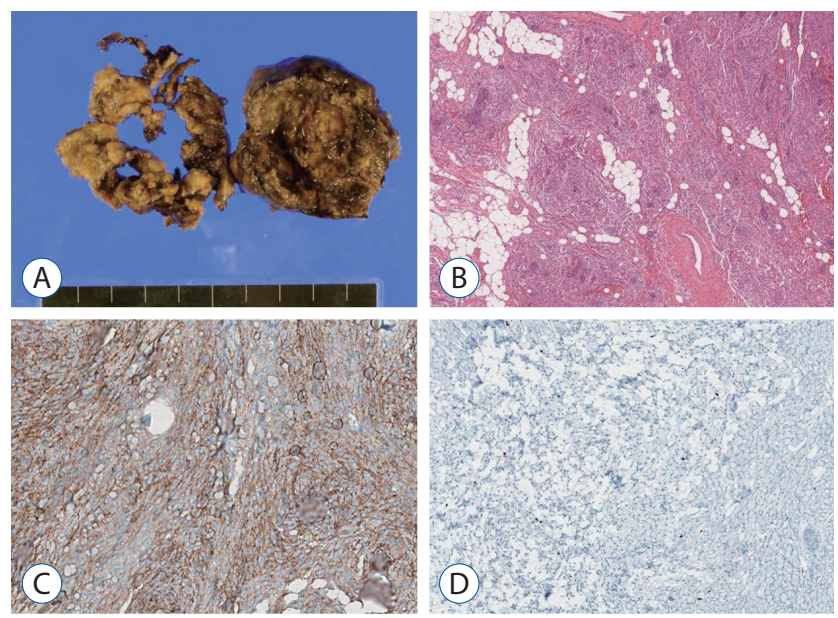

Fig. 3. A : Gross photo of the surgically resected specimen (formalin-fixed overnight). B : Representative microscopic image shows admixed meningioma tumor cells and fat component (hematoxylin \& eosin stain, $\times 40$ ). C : Membrane immunoreactivity of epithelial membrane antigen immunohistochemical stain observed (x100). D : Ki-67 immunohistochemical stain reveals low proliferative index of tumor cells (Positive in $5 \%, \times 100)$.
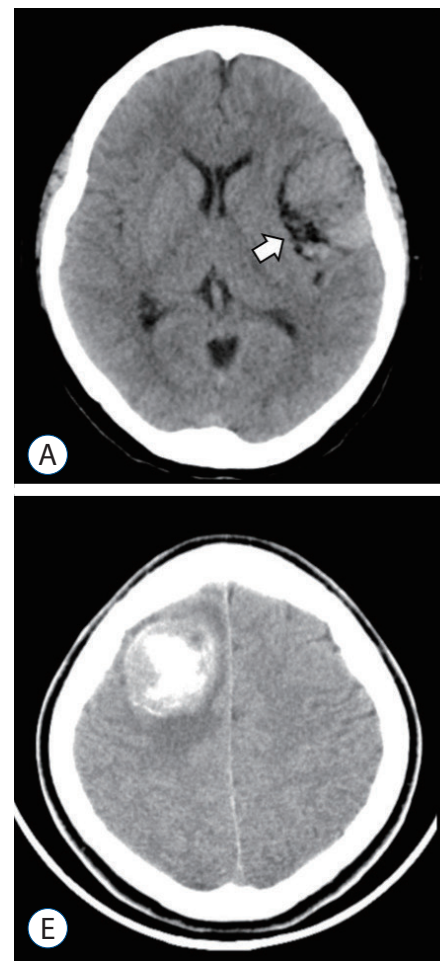
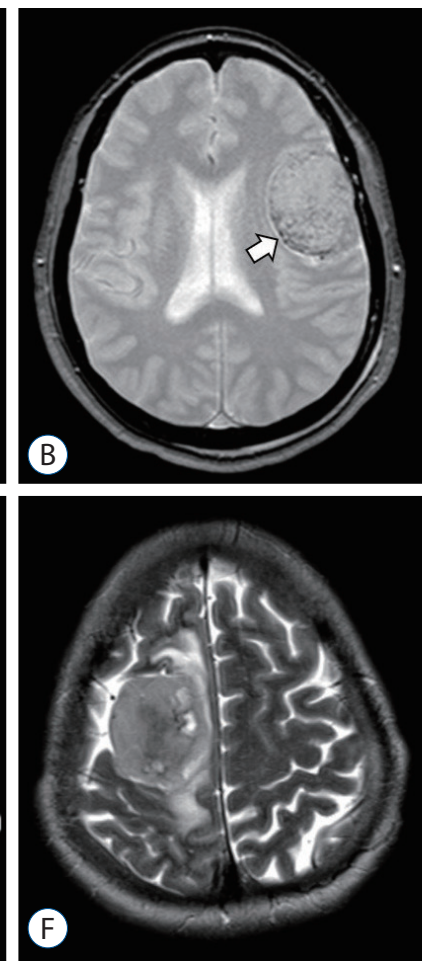
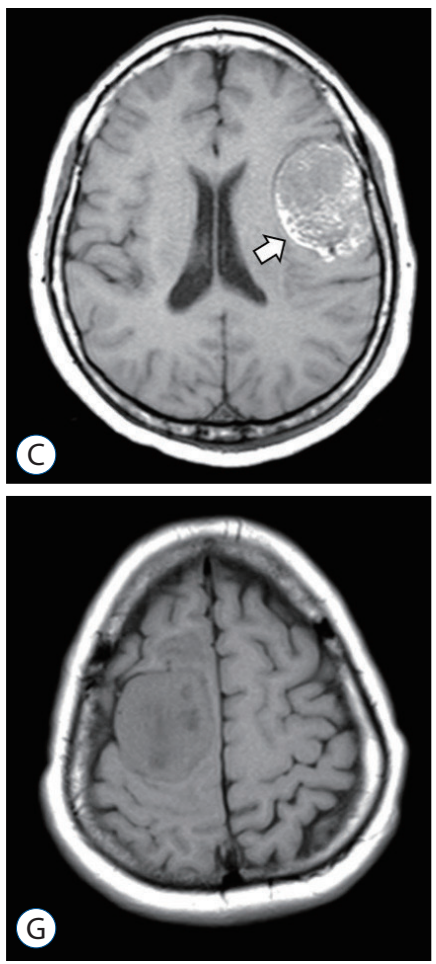
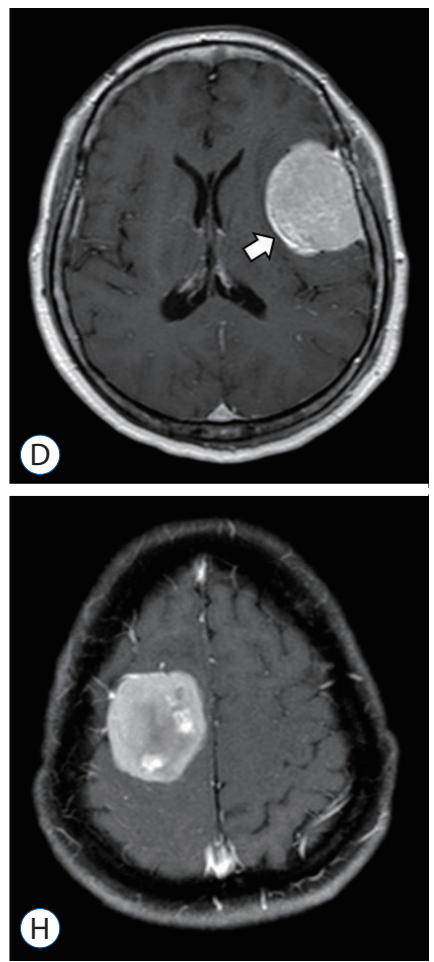

Fig. 2. A-D : Preoperative CT and MR images of lipomatous meningioma (case 1). White arrow suggesting the involvement of fatty components. E-H : Osseous meningioma (case 9). CT : computed tomography, MR: magnetic resonance. 
eration was noted within the lobules and in the surrounding stroma. Immunohistochemistry of the tumor cells showed strong membrane positivity to epithelial membrane antigen.

Other microscopic features showed only increased cellularity. There was no brain invasion, foci of spontaneous necrosis, uninterrupted patternless or sheet-like growth, prominent nucleoli, or small cells with a high nucleus/cytoplasm ratio. The mitotic count was 3 per 10 high power fields. The final pathologic diagnosis was metaplastic meningioma with rich fat component. The detailed pathological features are shown in Fig. 3.

\section{Illustrative case 2}

A 37-year-old woman suffering from a headache over several weeks presented generalized tonic-clonic seizure. Brain CT showed a $4.2 \mathrm{~cm}$ heterogeneously hyperdense lesion on the right frontal lobe suggesting calcification. Brain MR images also revealed heterogeneously high intensity on T1-gadolinium enhanced images (Fig. 2E-H). Preoperative radiological diagnosis was meningioma with dense calcification.

Frontal craniotomy was performed as usual, yellowish firm mass with dense calcification was revealed. The total removal of mass was accomplished, and the patient was discharged after seizure-free status. Histopathological examination showed that the tumor had diffuse osseous metaplasia with brain invasion. The tumor was finally diagnosed WHO grade I a metaplastic meningioma with osseous metaplasia.

\section{Summary of metaplastic meningioma cases}

The clinical, radiological, and histopathological details of all 11 patients are summarized in Table 1 . There were four male and seven female patients, with a mean long tumor diameter of $4.8 \mathrm{~cm}$ (range, 3.4-6.2) and mean age of 60 years (range, 24-65).

Detailed subclassification of the 11 metaplastic meningiomas showed that five cases were lipomatous, four osseous, and two xanthomatous subtypes. In our 15-year meningioma survey, lipomatous meningioma was the most common subclass of metaplastic meningioma. Cartilaginous subtype was also found in our patient population.

We found no common or unique radiological characteristics among all metaplastic meningiomas. However, obvious calcification on CT and marked fat signal intensity on MR images were detected in the osseous-type and lipomatous-

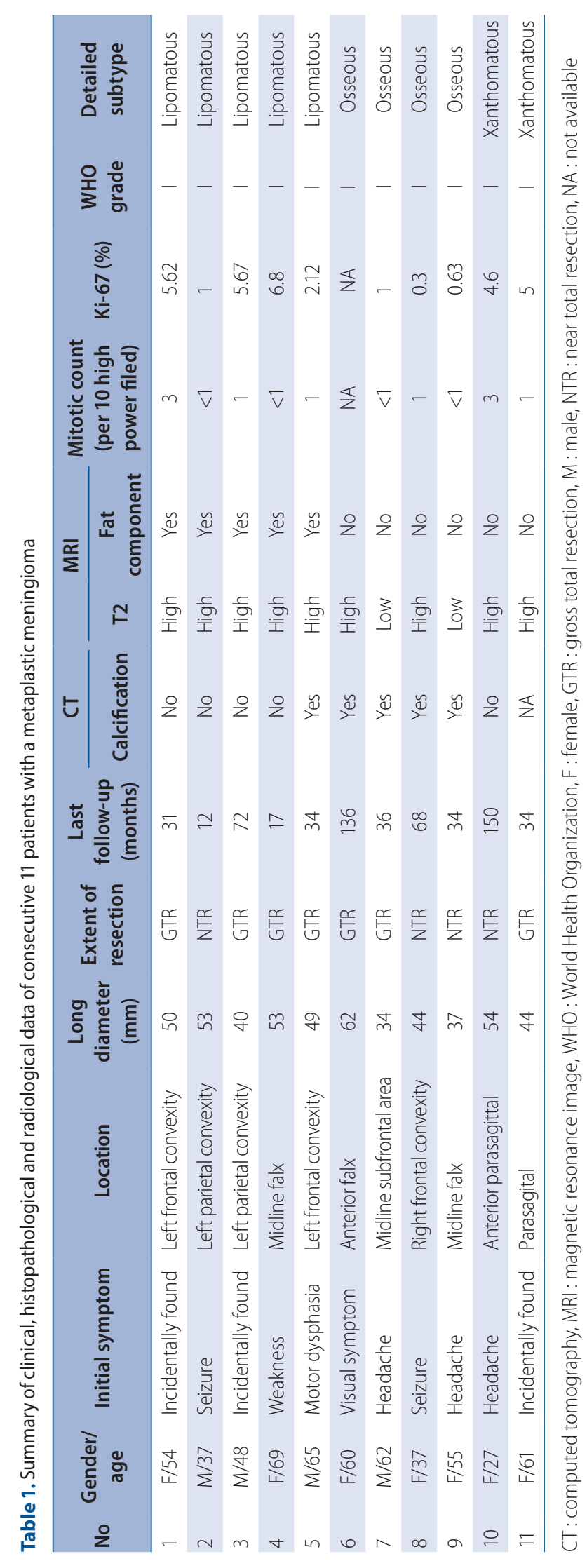


type metaplastic meningiomas, respectively.

\section{DISCUSSION}

Although meningioma are the most common primary extra-axial tumors, some subtypes are unfamiliar to many neurosurgeons. This is primarily due to their low incidence, especially since WHO grade I meningioma include nine subtypes, based on their histopathological features. Among these, metaplastic meningioma is among the rarest. In 2018, Zouaoui and colleagues $^{22)}$ reported a nationwide descriptive epidemiology of meningioma. In their 15-year study in France, the incidence of metaplastic meningioma was $0.04 \%$ (62 of 13038 newly diagnosed and histologically confirmed meningioma). Another epidemiologic study also showed the extreme rarity of metaplastic meningioma, with an incidence of $0.21 \%$ (15 of 7084 (cases) ${ }^{18)}$. Similarly, the proportion of metaplastic meningioma was $0.8 \%$ in our 15 -year surgical experience with meningiomas. Thus, our data reconfirm that it is among the rarest meningioma subtypes.

Another notable study finding was our initial evaluation of meningioma distribution according to WHO grade. During our 15-year study period, WHO grade I meningioma accounted for 1376 (75.9\%) cases : 354 (19.5\%) and 84 (4.6\%) cases were WHO grades II and III, respectively. The two epidemiologic studies described above reported $89.2 \%$ and $91.9 \%$ WHO grade I, 9.3\% and 5.2\% WHO grade II, and $1.5 \%$ and $2.9 \%$ WHO grade III meningioma, respectively ${ }^{20,22)}$. Thus, our incidence rates of WHO grades II and III meningiomas appear slightly higher than those reported previously. However, we must acknowledge several inherent study limitations. The study was retrospective and conducted at a single-center over 15 years. Possible selection bias was included due to the rarity and heterogeneity of metaplastic meningioma and the protocol did not reflect changes over time in the WHO classification criteria.

Metaplastic meningioma is referred to by several different names based on the presence of metaplastic changes to the dominant mesenchymal component : osseous, lipomatous, cartilaginous, myxoid and xanthomatous meninigoma ${ }^{21)}$. The term 'metaplastic' is used because the transformed neoplastic cells demonstrate the full histological characteristics of the cells they mimic $^{19)}$. Lipomatous metaplastic meningioma are made up of adipocyte resembling fatty cells with a signet-ring appearance ${ }^{3,6,211}$. Myxoid metaplastic meningioma are characterized by their excessive hyaluronic acid and chondroitin sulfate ${ }^{5,7,9,12}$. Osseous subtypes are confirmed by the presence of mature bone tissue and tumor cells in regions containing no psammoma bodies ${ }^{1,14,15,17,18)}$. A recent literature review by Choi et al. ${ }^{2)}$ described 26 metaplastic meningiomas, including 12 osseous subtypes, nine lipomatous subtypes, four xanthomatous subtypes, and one cartilaginous subtype. In our study, we were able to identify subtypes within the 11 metaplastic meningiomas, including nine lipomatous, four osseous, and two xanthomatous. No cartilaginous metaplastic meningioma was identified. Most metaplastic meningioma in our study were lipomatous or osseous metaplastic meningioma, similar to previous studies.

On radiological examination, the lipomatous type metaplastic meningioma demonstrated peculiar radiological features associated with a fatty component (Table 1, case 1 of Fig. 2). Although calcification is commonly observed in a meningioma, osseous type metaplastic meningioma had a distinct calcification on CT and MR images (Table 1, case 9 of Fig. 2). No other unique clinical characteristics of metaplastic meningioma were found.

The primary study purpose was to determine whether metaplastic meningiomas have unique clinical or radiological characteristics. We were unable to identify any unusual characteristics that might help distinguish metaplastic meningioma from other meningioma subtypes. However, toward further defining metaplastic meningioma, we note some common radiological and histological characteristics, such as the representative cases of lipomatous and osseous metaplastic meningioma. Despite the study limitations described above, we expect this study to confer a better understanding of the nature of these rare tumors.

\section{CONCLUSION}

We investigated a rare metaplastic meningioma subtype based on our 15-year surgical experience with meningiomas. Metaplastic meningiomas are classified based on their mesenchymal components, some of which have unique radiological characteristics. Further investigation will be necessary to describe the nature of these rare tumors more clearly. 


\section{CONFLICTS OF INTEREST}

No potential conflict of interest relevant to this article was reported.

\section{INFORMED CONSENT}

This type of study does not require informed consent.

\section{AUTHOR CONTRIBUTIONS}

\author{
Conceptualization : TK, JWK \\ Data curation : TK, SYJ, HK, KMK, SHC, SHP \\ Formal analysis : TK, JWK, SHP \\ Methodology: YHK, CKP, JWK \\ Project administration : JWK \\ Visualization : TK \\ Writing - original draft : TK, JWK \\ Writing - review \& editing: YHK, CKP, JWK
}

\section{ORCID}

$\begin{array}{ll}\text { Taehoon Kim } & \text { https://orcid.org/0000-0001-7157-2119 } \\ \text { Jin Wook Kim } & \text { https://orcid.org/0000-0002-3773-3940 } \\ \text { So Young Ji } & \text { https://orcid.org/0000-0003-0939-3130 } \\ \text { Ho Kang } & \text { https://orcid.org/0000-0003-2143-410X } \\ \text { Kyung-Min Kim } & \text { https://orcid.org/0000-0002-1150-3338 } \\ \text { Yong Hwy Kim } & \text { https://orcid.org/0000-0001-9009-4191 } \\ \text { Chul-Kee Park } & \text { https://orcid.org/0000-0002-2350-9876 } \\ \text { Seung Hong Choi } & \text { https://orcid.org/0000-0002-0412-2270 } \\ \text { Sung-Hye Park } & \text { https://orcid.org/0000-0002-8681-1597 }\end{array}$

\section{References}

1. Barresi V, Caffo M, leni A, Alafaci C, Tuccari G : Osteoblastic meningiomas: clinico-pathological and immunohistochemical features of an uncommon variant. J Neurooncol 105 : 225-232, 2011

2. Choi YH, Choi CY, Lee CH, Koo HW, Chang SH : Metaplastic meningioma overspreading the cerebral convexity. Brain Tumor Res Treat 6 : 97-100, 2018

3. Colnat-Coulbois S, Kremer S, Weinbreck N, Pinelli C, Auque J : Lipoma- tous meningioma: report of 2 cases and review of the literature. Surg Neurol 69 : 398-402; discussion 402, 2008

4. Danisman MC, Kelesoglu KS, Sivri M, Koplay M, Paksoy Y : Microcystic meningioma: difficulties in diagnosis and magnetic resonance imaging findings. Acta Neurol Belg 117 : 745-747, 2017

5. Ersoz S, Yilmaz ZS, Eyuboglu I, Yazar U : Xanthomatous meningioma: a case report. Turk Neurosurg 29 : 141-144, 2019

6. Gasparinho MG, Ferreira M, Lavrador JP, Livraghi $\mathrm{S}$ : Revisiting lipomatous meningioma: a case report and review of an unusual entity. Int J Surg Pathol 23 : 399-403, 2015

7. Ikota $H$, Nakazato $Y$ : A case of metaplastic meningioma with extensive xanthomatous change. Neuropathology 28 : 422-426, 2008

8. Indiran V : Intracranial ossified metaplastic meningioma: unusual cause of headache. J Neurosci Rural Pract 8 : 653, 2017

9. Ishida M, Fukami T, Nitta N, Iwai M, Yoshida K, Kagotani A, et al. : Xanthomatous meningioma: a case report with review of the literature. Int J Clin Exp Pathol 6 : 2242-2246, 2013

10. Kang H, Kim JW, Se YB, Dho YS, Choi SH, Park SH : Sclerosing meningioma : radiological and clinical characteristics of 21 cases. J Korean Neurosurg Soc 59 : 584-589, 2016

11. Kim NR, Yee GT, Cho HY : Crush cytology of secretory meningioma: a case report. Brain Tumor Res Treat 3 : 147-150, 2015

12. Krisht KM, Altay T, Couldwell WT : Myxoid meningioma: a rare metaplastic meningioma variant in a patient presenting with intratumoral hemorrhage. J Neurosurg 116 : 861-865, 2012

13. Kunimatsu A, Kunimatsu N, Kamiya K, Katsura M, Mori H, Ohtomo K : Variants of meningiomas: a review of imaging findings and clinical features. Jpn J Radiol 34 : 459-469, 2016

14. Mannoji C, Koda M, Murakami M, Kubosawa H, Yamazaki M, Okawa A, et al. : Osseous metaplastic meningioma in the thoracic spine mimicking osteosarcoma: a case report. Spine (Phila Pa 1976) 38 : E632-E634, 2013

15. Murakami T, Tanishima S, Takeda C, Kato S, Nagashima H : Ossified metaplastic spinal meningioma without psammomatous calcification: a case report. Yonago Acta Med 62 : 232-235, 2019

16. Paek SH, Kim SH, Chang KH, Park CK, Kim JE, Kim DG, et al. : Microcystic meningiomas: radiological characteristics of 16 cases. Acta Neurochir (Wien) 147 : 965-972; discussion 972, 2005

17. Salunke P, Aggarwal A, Futane S, Nada R, Gochhait D : Osteoblastic meningioma with turtle shell: different entity from calcified meningioma. Asian J Neurosurg 11 : 450, 2016

18. Somerset HL, Kleinschmidt-DeMasters BK, Rubinstein D, Breeze RE : Osteochondroma of the convexity: pathologic-neuroimaging correlates of a lesion that mimics high-grade meningioma. J Neurooncol 98 : 421 426, 2010

19. Tang $H$, Sun $H$, Chen $H$, Gong $Y$, Mao Y, Xie Q, et al. : Clinicopathological analysis of metaplastic meningioma: report of 15 cases in Huashan Hospital. Chin J Cancer Res 25 : 112-118, 2013

20. Wang DJ, Xie Q, Gong Y, Mao Y, Wang Y, Cheng HX, et al. : Histopathological classification and location of consecutively operated meningiomas at a single institution in China from 2001 to 2010. Chin Med J 
Meningioma of Metaplasia | Kim T, et al.

(Engl) $126:$ 488-493, 2013

21. Yüksel MO, Gürbüz MS, Tanriverdi O, Özmen SA : Lipomatous meningioma: a rare subtype of benign metaplastic meningiomas. J Neurosci Rural Pract 8 : 140-142, 2017
22. Zouaoui S, Darlix A, Rigau V, Mathieu-Daudé $H$, Bauchet $F$, Bessaoud F, et al. : Descriptive epidemiology of 13,038 newly diagnosed and histologically confirmed meningiomas in France: 2006-2010. Neurochirurgie 64 : 15-21, 2018 